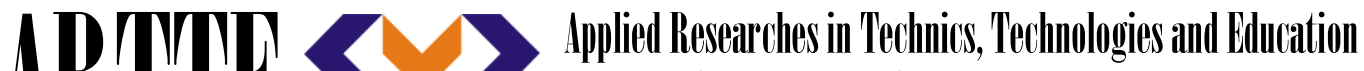 Journal of the Faculty of Technics and Technologies, Trakia University https://sites.google.com/a/trakia-uni.bg/artte/
}

\section{STUDY OF WEED FLORA OF MAIZE CROPS IN THE NISAVA DISTRICT}

\author{
Jelica Živić, Milić Vojinović, Ivica Stančić, Saša Petrović \\ College of Agriculture and Food Technology, Prokuplje, Serbia \\ Ćirila i Metodija br.1,18400 Prokuplje, Serbia \\ phone: +38127/324-311, email: vpps@beotel.rs
}

\begin{abstract}
The damage from the present weeds in maize crop is shown by the reduction of yield in almost every year and their suppression is completely economically justified. The right selection of herbicides mostly depends on the presence of dominant weed species and the time of application. The paper presents the distribution and existence of economically harmful, invasive and quarantine weed species (Abutilon theophrasti, Agropyrum repens, Amaranthus retroflexus, Ambrosia artemisiifolia, Capsela bursa-pastoris, Chenopodium album, Chenopodium hybridum, Cirsium arvense, Convolvulus arvensis, Datura stramonium, Avena fatua, Calystegia sepium, ...) on ten locations of maize crops in the Nisava district. Estimation of the species presence was done in two shootings (May and August) on scale 1-4 (1- species appears individually and occupy up to 5\% of the surface, 2 - appears and occupies 5-25\% of the surface, 3 - appears often and occupies $25-50 \%$ of the surface, 4 - the species prevails over the cultivated plant and occupies over $50 \%$ of the surface).

Keywords: weed flora, maize crop, existence, distribution.
\end{abstract}

\section{INTRODUCTION}

The composition of the cow's flora in one habitat depends primarily on the characteristics of climate and soil, crop types, applied agro-technology (fertilization, soil treatment, fertilization, herbicide application, etc.), as with other factors. Herbicides usage is one of the factors that has significantly affected the change of the composition of weed communities in recent years [4]. Long-term usage of herbicides with similar or the same active substance has led to a greater presence of more resistant weeds [1].The analysis of the number and representation of the most important weed species enables the application of adequate measures in the protection of bred crops and the control of new species of weed community. Also, results of the researches can be used as one of the ways of effective control of weed vegetation in the concept of sustainable agriculture.

Former researches of presence and representation of weed species in maize crop at Nisava district are very poor. Because of that, results of these researches can be used proper selection of control measures as well as for the monitoring of weed species in these areas.

\section{METHODS}

The shooting had included 10 sites in the Nisava district, plots under the maize crop. The presence and representation of 25 weeds were observed. The applied method of assessing the representation of mapped species was as follows:

- method of evaluation: visual

- evaluation time: 2 measurements per season

- the size of the assessed area: 0.5 ha, 1 parcel, $100 \mathrm{~m}$ bound to the npp.

- number of recordings per crop: 3.The representation of the mapped species was done on scale 1-4 (1 - the species is shown individually and occupies up to $5 \%$ of the surface; 2 shown and occupies $5-25 \%$ of the surface; 3 - shown often $25-50 \%$ of the surface; 4 - the 
species prevails over the cultivated plant and occupies over $50 \%$ of the area [2,5].The plant crop phase (according to the BBCH scale) is equal as phase of more than $50 \%$ of the plants. Phase of the weed (PW): P - from cotyledons up to 5.6 plant leaf, $V$ - plant, $G$ - phase of fertility.

\section{EXPERIMENTAL RESULTS}

The results of phytocoenological shootings are shown in the tables.

Table 1. Distribution of weed species in maize crop (the first shooting on first five locations)

\begin{tabular}{|c|c|c|c|c|c|c|c|c|c|c|}
\hline $\begin{array}{l}\text { Main informations about } \\
\text { date and shooting place }\end{array}$ & \multicolumn{2}{|c|}{ 1. shooting } & \multicolumn{2}{|c|}{ 1. shooting } & \multicolumn{2}{|c|}{ 1. shooting } & \multicolumn{2}{|c|}{ 1. shooting } & \multicolumn{2}{|c|}{ 1. shooting } \\
\hline Date of shooting & \multicolumn{2}{|c|}{24.05.} & \multicolumn{2}{|c|}{24.05.} & \multicolumn{2}{|c|}{24.05.} & \multicolumn{2}{|c|}{24.05.} & \multicolumn{2}{|c|}{16.05} \\
\hline Place (nearest inhabited town): & \multicolumn{2}{|c|}{ Bovan } & \multicolumn{2}{|c|}{ Subotinac } & \multicolumn{2}{|c|}{ Glogovica } & \multicolumn{2}{|c|}{ Mozgovo } & \multicolumn{2}{|c|}{ Rutevac } \\
\hline $\begin{array}{l}\text { Coordinates (GPS) } \\
\text { W: }\end{array}$ & \multicolumn{2}{|c|}{ [7556288] } & \multicolumn{2}{|c|}{$[7554726]$} & \multicolumn{2}{|c|}{$[7559970]$} & \multicolumn{2}{|c|}{$[7553054]$} & \multicolumn{2}{|c|}{ [7550308] } \\
\hline E: & \multicolumn{2}{|c|}{ [4830763] } & \multicolumn{2}{|c|}{ [4831686] } & \multicolumn{2}{|c|}{ [4820009] } & \multicolumn{2}{|c|}{ [4834752] } & \multicolumn{2}{|c|}{ [4828595] } \\
\hline A: & \multicolumn{2}{|c|}{$[215 \pm 4]$} & {$[257$} & & {$[190$} & & {$[328$} & & {$[174$} & \\
\hline Grown plant: & maiz & & $\mathrm{ma}$ & & $\mathrm{ma}$ & & $\mathrm{ma}$ & & $\mathrm{ma}$ & \\
\hline $\begin{array}{l}\text { Phase of grown plant } \\
\text { (accordnig to } \mathrm{BBCH} \text { scale): }\end{array}$ & 1 & & 1 & & 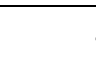 & & 1 & & 1 & \\
\hline $\begin{array}{l}\text { Herbicide usage: } \\
\text { (enroll yes, no or unknown) }\end{array}$ & unkno & & unkn & & $y$ & & unkn & & nc & \\
\hline Information & f repres & entati & n of we & d fro & scale a & $1-4$ & nase of & eed & & \\
\hline Weed specie & $\begin{array}{c}\text { Repre. } \\
\text { mark }\end{array}$ & PW & $\begin{array}{l}\text { Repre. } \\
\text { Mark }\end{array}$ & PW & $\begin{array}{l}\text { Repre. } \\
\text { mark }\end{array}$ & PW & $\begin{array}{c}\text { Repre. } \\
\text { mark }\end{array}$ & PW & $\begin{array}{c}\text { Repre. } \\
\text { mark }\end{array}$ & PW \\
\hline 1. Abutilon theophrasti & & & & & & & & & & \\
\hline 2. Agropyrum repens & 1 & $\mathrm{~V}$ & 1 & $\mathrm{~V}$ & 1 & $\mathrm{~V}$ & & & 1 & $\mathrm{~V}$ \\
\hline 3. Amaranthus retroflexus & & & & & & & & & 1 & $\mathrm{~V}$ \\
\hline 4. Amaranthus hybridus & & & & & & & & & & \\
\hline 5. Ambrosia artemisiifolia & & & & & & & & & & \\
\hline 6. Asclepias syriaca & & & & & & & & & & \\
\hline 7. Avena fatua & & & & & & & & & & \\
\hline 8. Calystegia sepium & 1 & $\mathrm{~V}$ & 1 & $\mathrm{v}$ & 1 & $\mathrm{~V}$ & 1 & $\mathrm{~V}$ & & \\
\hline 9. Cannabis sativa & & & & & & & & & & \\
\hline 10. Cirsium arvense & 2 & $\mathrm{~V}$ & 1 & $\mathrm{v}$ & 1 & $\mathrm{v}$ & 1 & $\mathrm{~V}$ & 1 & $\mathrm{v}$ \\
\hline 11. Chenopodium album & 1 & $\mathrm{~V}$ & 1 & $\mathrm{v}$ & & & 1 & $\mathrm{~V}$ & 1 & $\mathrm{~V}$ \\
\hline 12. Chenopodium hybridu. & & & & & 1 & $\mathrm{p}$ & & & & \\
\hline 13. Convolvulus arvensis & 1 & $\mathrm{~V}$ & 1 & $\mathrm{v}$ & 1 & $\mathrm{~V}$ & 1 & $\mathrm{~V}$ & 1 & $\mathrm{~V}$ \\
\hline 14. Cuscuta spp. & & & & & & & & & & \\
\hline 15. Cynodon dactylon & 2 & V & 1 & V & & & 1 & $\mathrm{~V}$ & 1 & $\mathrm{~V}$ \\
\hline 16. Datura stramonium & & & & & & & & & & \\
\hline 17. Erigeron canadensis & & & & & & & & & & \\
\hline 18. Galium aparine & & & & & & & & & & \\
\hline 19. Helianthus tuberosus & & & & & & & & & & \\
\hline 20. Iva xanthifolia & & & & & & & & & & \\
\hline 21. Lactuca serriola & & & & & & & & & & \\
\hline 22. Orobanche spp. & & & & & & & & & & \\
\hline 23. Sonchus arvensis & & & & & & & & & & \\
\hline 24. Sorghum halepense & & & & & 1 & $\mathrm{~V}$ & & & 2 & $p-v$ \\
\hline 25. Xanthium strumarium & 1 & $P$ & & & & & & & & \\
\hline
\end{tabular}


Table 2. Distribution of weed species in maize crop (the first shooting on second five locations)

\begin{tabular}{|c|c|c|c|c|c|c|c|c|c|c|}
\hline $\begin{array}{l}\text { Main informations about } \\
\text { date and shooting place }\end{array}$ & \multicolumn{2}{|c|}{ 1. shooting } & \multicolumn{2}{|c|}{ 1. shooting } & \multicolumn{2}{|c|}{ 1. shooting } & \multicolumn{2}{|c|}{ 1. shooting } & \multicolumn{2}{|c|}{ 1. shooting } \\
\hline Date of shooting & \multicolumn{2}{|c|}{16.05} & \multicolumn{2}{|c|}{16.05} & \multicolumn{2}{|c|}{16.05.} & \multicolumn{2}{|c|}{10.05} & \multicolumn{2}{|c|}{10.05} \\
\hline Place (nearest inhabited town): & \multicolumn{2}{|c|}{ Donja Trnava } & \multicolumn{2}{|c|}{ Drazevac } & \multicolumn{2}{|c|}{ Deligrad } & \multicolumn{2}{|c|}{ Moravac } & \multicolumn{2}{|c|}{ Nozrina } \\
\hline $\begin{array}{l}\text { Coordinates (GPS) } \\
\text { W: }\end{array}$ & \multicolumn{2}{|c|}{ [7565001] } & \multicolumn{2}{|c|}{ [7565054] } & \multicolumn{2}{|c|}{ [7547957] } & \multicolumn{2}{|c|}{ [7558041] } & \multicolumn{2}{|c|}{ [7558520] } \\
\hline E: & \multicolumn{2}{|c|}{ [4807938] } & \multicolumn{2}{|c|}{ [4811595] } & \multicolumn{2}{|c|}{ [4830128] } & \multicolumn{2}{|c|}{ [4816553] } & \multicolumn{2}{|c|}{ [4815400] } \\
\hline A: & \multirow{2}{*}{\multicolumn{2}{|c|}{$\begin{array}{l}{[194 \pm 5]} \\
\text { maize }\end{array}$}} & {$[180=$} & & {$[176$} & & {$[176$} & & {$[182$} & \\
\hline Grown plant: & & & maiz & & $\mathrm{ma}$ & & mai & & mai & \\
\hline $\begin{array}{l}\text { Phase of grown plant } \\
\text { (accordnig to BBCH scale): }\end{array}$ & & & 1 & & 1 & & 1 & & 1 & \\
\hline $\begin{array}{l}\text { Herbicide usage: } \\
\text { (enroll yes, no or unknown) }\end{array}$ & unk & & unknc & & unkn & & unkn & & unkn & \\
\hline Informatio & f repres & ntatiol & f weed & $\overline{\text { om s }}$ & ale and & $-4 \mathrm{ph}$ & se of $w$ & & & \\
\hline Weed specie & $\begin{array}{l}\text { Repre. } \\
\text { mark }\end{array}$ & PW & $\begin{array}{l}\text { Repre. } \\
\text { mark }\end{array}$ & PW & $\begin{array}{c}\text { Repre. } \\
\text { mark }\end{array}$ & PW & $\begin{array}{l}\text { Repre. } \\
\text { mark }\end{array}$ & PW & $\begin{array}{l}\text { Repre. } \\
\text { mark }\end{array}$ & PW \\
\hline 1. Abutilon theophrasti & 1 & $p-v$ & 1 & $p-v$ & & & & & & \\
\hline 2. Agropyrum repens & & & & & 1 & $\mathrm{~V}$ & & & & \\
\hline 3. Amaranthus retroflexus & & & & & 1 & $\mathrm{~V}$ & & & & \\
\hline 4. Amaranthus hybridus & & & & & & & 1 & $p$ & 1 & $p$ \\
\hline 5. Ambrosia artemisiifolia & & & & & & & & & & \\
\hline 6. Asclepias syriaca & & & & & & & & & & \\
\hline 7. Avena fatua & & & & & & & & & & \\
\hline 8. Calystegia sepium & & & & & & & 1 & $p-v$ & 1 & $p-v$ \\
\hline 9. Cannabis sativa & 1 & $p-v$ & 1 & $p-v$ & & & & & & \\
\hline 10. Cirsium arvense & 1 & $p-v$ & 1 & $p-v$ & 1 & $\mathrm{~V}$ & & & & \\
\hline 11. Chenopodium album & & & & & 1 & V & & & & \\
\hline 12. Chenopodium hybridum & & & & & & & 1 & $p-v$ & 1 & $p-v$ \\
\hline 13. Convolvulus arvensis & & & & & 1 & $\mathrm{~V}$ & 1 & $p-v$ & 1 & $p-v$ \\
\hline 14. Cuscuta spp. & 1 & $p-v$ & 1 & $p-v$ & & & & & & \\
\hline 15. Cynodon dactylon & & & & & 1 & V & & & & \\
\hline 16. Datura stramonium & & & & & & & & & & \\
\hline 17. Erigeron canadensis & & & & & & & & & & \\
\hline 18. Galium aparine & & & & & & & & & & \\
\hline 19. Helianthus tuberosus & & & & & & & & & & \\
\hline 20. Iva xanthifolia & & & & & & & & & & \\
\hline 21. Lactuca serriola & & & & & & & & & & \\
\hline 22. Orobanche spp. & 1 & $p-v$ & 1 & $p-v$ & & & & & & \\
\hline 23. Sonchus arvensis & 1 & $p-v$ & 1 & $p-v$ & & & & & & \\
\hline 24. Sorghum halepense & & & & & 1 & $\mathrm{P}$ & & & & \\
\hline 25. Xanthium strumarium & & & & & & & & & & \\
\hline
\end{tabular}

Table 1 shows the presence and number of recorded weeds in phase of the adult plant (V) at five locations. Maze crop was in phase 1. Present weeds were estimated with mark 1 at all localities. At location Bovan weeds Cirsium arvense and Cynodon dactylon occupied 5-25\% of maize crop (mark 2).

In Table 2 the presence and the number of recorded weeds at 10 and 16 of May were estimated, and the weeds were in the to 5.6 plant leaf to the adult plant and the phase of the growth of corn 1, at the five selected locations. At all localities weeds were present in most of the evaluation grades 1. At the locality of Deligrad it was noted the biggest number of weed species. 
Table 3. Distribution of weed species in maize crop (the second shooting on first five locations)

\begin{tabular}{|c|c|c|c|c|c|c|c|c|c|c|}
\hline $\begin{array}{l}\text { Main informations about } \\
\text { date and shooting place }\end{array}$ & \multicolumn{2}{|c|}{ 2. shooting } & \multicolumn{2}{|c|}{ 2. shooting } & \multicolumn{2}{|c|}{ 2. shooting } & \multicolumn{2}{|c|}{ 2. shooting } & \multicolumn{2}{|c|}{ 2. shooting } \\
\hline Date of shooting & \multicolumn{2}{|c|}{09.08.} & \multicolumn{2}{|c|}{22.08.} & \multicolumn{2}{|c|}{09.08.} & \multicolumn{2}{|c|}{22.08.} & \multicolumn{2}{|c|}{22.08.} \\
\hline $\begin{array}{l}\text { Place (nearest inhabited } \\
\text { town): }\end{array}$ & \multicolumn{2}{|c|}{ Bovan } & \multicolumn{2}{|c|}{ Subotinac } & \multicolumn{2}{|c|}{ Glogovica } & \multicolumn{2}{|c|}{ Mozovo } & \multicolumn{2}{|c|}{ Rutevac } \\
\hline Grown plant: & \multicolumn{2}{|c|}{ maize } & \multicolumn{2}{|c|}{ maize } & \multicolumn{2}{|c|}{ maize } & \multicolumn{2}{|c|}{ maize } & \multicolumn{2}{|c|}{ maize } \\
\hline $\begin{array}{l}\text { Phase of grown plant } \\
\text { (accordnig to BBCH scale): }\end{array}$ & \multicolumn{2}{|c|}{8} & \multicolumn{2}{|c|}{8} & \multicolumn{2}{|c|}{8} & \multicolumn{2}{|c|}{8} & \multicolumn{2}{|c|}{8} \\
\hline $\begin{array}{l}\text { Herbicide usage: } \\
\text { (enroll yes, no or unknown) }\end{array}$ & \multicolumn{2}{|c|}{ unknown } & \multicolumn{2}{|c|}{ unknown } & unkn & & unkn & & unknc & \\
\hline Informatio & of repr & senta & n of we & d fror & cale an & $1-4$ & ase of $v$ & & & \\
\hline Weed specie & $\begin{array}{l}\text { Repre. } \\
\text { mark }\end{array}$ & PW & $\begin{array}{c}\text { Repre. } \\
\text { mark }\end{array}$ & PW & $\begin{array}{l}\text { Repre. } \\
\text { mark }\end{array}$ & PW & $\begin{array}{c}\text { Repre. } \\
\text { mark }\end{array}$ & PW & $\begin{array}{l}\text { Repre. } \\
\text { mark }\end{array}$ & PW \\
\hline 1. Abutilon theophrasti & & & & & 2 & $\mathrm{~V}$ & & & & \\
\hline 2. Agropyrum repens & 2 & $\mathrm{~V}$ & 2 & $\mathrm{G}$ & 2 & $G$ & 2 & $\mathrm{G}$ & 3 & $G$ \\
\hline 3. Amaranthus retroflexus & 2 & $\mathrm{G}$ & 3 & $\mathrm{G}$ & & & 3 & $\mathrm{G}$ & 3 & $\mathrm{G}$ \\
\hline 4. Amaranthus hybridus & & & & & & & & & 1 & $\mathrm{G}$ \\
\hline 5. Ambrosia artemisiifolia & & & 1 & V-G & & & & & & \\
\hline 6. Asclepias syriaca & & & & & & & & & & \\
\hline 7. Avena fatua & & & & & 3 & V & & & & \\
\hline 8. Calystegia sepium & 3 & $\mathrm{~V}$ & & & & & & & & \\
\hline 9. Cannabis sativa & & & & & 1 & $\mathrm{~V}$ & & & & \\
\hline 10. Cirsium arvense & 1 & $\mathrm{~V}$ & 3 & $\mathrm{G}$ & 1 & $\mathrm{G}$ & 3 & G & 2 & $\mathrm{G}$ \\
\hline 11. Chenopodium album & 1 & $G$ & 3 & $\mathrm{G}$ & & & 3 & $\mathrm{G}$ & 3 & $G$ \\
\hline 12. Chenopodium hybridu. & & & & & 2 & $\mathrm{~V}$ & & & & \\
\hline 13. Convolvulus arvensis & 2 & $\mathrm{~V}$ & 1 & $\mathrm{~V}$ & & & 2 & $\mathrm{~V}$ & 2 & V \\
\hline 14. Cuscuta spp. & & & & & 1 & $\mathrm{G}$ & & & & \\
\hline 15. Cynodon dactylon & 1 & $\mathrm{G}$ & 3 & $\mathrm{G}$ & & & 2 & G & 2 & $\mathrm{G}$ \\
\hline 16. Datura stramonium & & & 1 & $\mathrm{~V}$ & 1 & V & & & & \\
\hline 17. Erigeron canadensis & 1 & $\mathrm{~V}$ & & & & & 1 & $\mathrm{~V}$ & & \\
\hline 18. Galium aparine & & & & & & & & & & \\
\hline 19. Helianthus tuberosus & & & & & & & & & & \\
\hline 20. Iva xanthifolia & & & & & & & & & & \\
\hline 21. Lactuca serriola & & & & & & & & & 1 & $\mathrm{~V}$ \\
\hline 22. Orobanche spp. & & & & & & & & & & \\
\hline 23. Sonchus arvensis & & & & & & & & & & \\
\hline 24. Sorghum halepense & & & 1 & $\mathrm{G}$ & 1 & $\mathrm{~V}$ & 2 & $\mathrm{G}$ & & \\
\hline 25. Xanthium strumarium & 1 & V & 1 & V & & & & & & \\
\hline
\end{tabular}

Table 3 shows data from second shooting of weed distribution at 9 and 22 of August, at first five locations. Most of the weeds were in G phase (phase of fertility), while maize plants were in phase 8 (according to BBCH scale). Weed plants were seen in almost all locations, where marks were from 1 to 3 . Weed species Agropyrum repens, Amaranthus retroflexus, Cirsium arvense and Chenopodium album were present at all locations with representation mark from 2 to 3. 
Table 4. Distribution of weed species in maize crop (the second shooting on second five locations)

\begin{tabular}{|c|c|c|c|c|c|c|c|c|c|c|}
\hline $\begin{array}{l}\text { Main informations about } \\
\text { date and shooting place }\end{array}$ & \multicolumn{2}{|c|}{ 2. shooting } & \multicolumn{2}{|c|}{ 2. shooting } & \multicolumn{2}{|c|}{ 2. shooting } & \multicolumn{2}{|c|}{ 2. shooting } & \multicolumn{2}{|c|}{ 2.shooting } \\
\hline Date of shooting & \multicolumn{2}{|c|}{ 09.08.05. } & \multicolumn{2}{|c|}{ 09.08.05. } & \multicolumn{2}{|c|}{ 22.08.05 } & \multicolumn{2}{|c|}{22.08 .05} & \multicolumn{2}{|c|}{22.08 .05} \\
\hline Place (nearest inhabited town): & \multicolumn{2}{|c|}{ D.Trnava } & \multicolumn{2}{|c|}{ Drazevac } & \multicolumn{2}{|c|}{ Deligrad } & \multicolumn{2}{|c|}{ Moravac } & \multicolumn{2}{|c|}{ Nozrina } \\
\hline Grown plant: & \multicolumn{2}{|c|}{ maize } & \multicolumn{2}{|c|}{ maize } & \multicolumn{2}{|c|}{ maize } & \multicolumn{2}{|c|}{ maize } & \multicolumn{2}{|c|}{ maize } \\
\hline $\begin{array}{l}\text { Phase of grown plant } \\
\text { (accordnig to BBCH scale): }\end{array}$ & \multicolumn{2}{|c|}{8} & \multicolumn{2}{|c|}{8} & \multicolumn{2}{|c|}{8} & \multicolumn{2}{|c|}{8} & \multicolumn{2}{|c|}{8} \\
\hline $\begin{array}{l}\text { Herbicide usage: } \\
\text { (enroll yes, no or unknown) }\end{array}$ & \multicolumn{2}{|c|}{ unknown } & \multicolumn{2}{|c|}{ unknown } & unkn & & unk & & unk & \\
\hline Informatio & of repres & ntat & of wee & from & ale anc & $-4 p$ & se of $v$ & & & \\
\hline Weed specie & $\begin{array}{l}\text { Repre. } \\
\text { mark }\end{array}$ & PW & $\begin{array}{l}\text { Repre. } \\
\text { Mark }\end{array}$ & PW & $\begin{array}{l}\text { Repre. } \\
\text { mark }\end{array}$ & PW & $\begin{array}{l}\text { Repre. } \\
\text { mark }\end{array}$ & PW & $\begin{array}{l}\text { Repre. } \\
\text { mark }\end{array}$ & PW \\
\hline 1. Abutilon theophrasti & & & 1 & $\mathrm{~V}$ & & & & & & \\
\hline 2. Agropyrum repens & 2 & $\mathrm{~V}$ & 2 & $\mathrm{~V}$ & 2 & $\mathrm{G}$ & 2 & $\mathrm{~V}$ & 2 & $\mathrm{G}$ \\
\hline 3. Amaranthus retroflexus & 2 & $\mathrm{G}$ & 2 & $\mathrm{~V}$ & 2 & $\mathrm{G}$ & 3 & $\mathrm{G}$ & 2 & $\mathrm{G}$ \\
\hline 4. Amaranthus hybridus & & & 2 & $\mathrm{~V}$ & & & & & & \\
\hline 5. Ambrosia artemisiifolia & & & & & 1 & $\mathrm{~V}$ & & & & \\
\hline 6. Asclepias syriaca & & & & & & & & & & \\
\hline 7. Avena fatua & & & & & & & & & & \\
\hline 8. Calystegia sepium & 2 & $\mathrm{~V}$ & 2 & $\mathrm{~V}$ & 2 & $\mathrm{~V}$ & 2 & $\mathrm{~V}$ & 2 & $\mathrm{~V}$ \\
\hline 9. Cannabis sativa & & & & & & & & & & \\
\hline 10. Cirsium arvense & 2 & $\mathrm{~V}$ & 3 & $\mathrm{~V}$ & 2 & $\mathrm{G}$ & 2 & $\mathrm{~V}$ & 2 & V \\
\hline 11. Chenopodium album & 2 & $\mathrm{G}$ & 3 & $\mathrm{~V}$ & 2 & $\mathrm{G}$ & 2 & $\mathrm{G}$ & 3 & $\mathrm{G}$ \\
\hline 12. Chenopodium hybridum & & & & & & & & & & \\
\hline 13. Convolvulus arvensis & 2 & $\mathrm{~V}$ & 1 & $\mathrm{~V}$ & 2 & $\mathrm{~V}$ & 2 & $\mathrm{~V}$ & 2 & $\mathrm{~V}$ \\
\hline 14. Cuscuta spp. & & & & & & & & & & \\
\hline 15. Cynodon dactylon & 2 & $\mathrm{G}$ & 3 & $\mathrm{~V}$ & 2 & $G$ & 2 & $\mathrm{G}$ & 2 & $\mathrm{G}$ \\
\hline 16. Datura stramonium & & & 1 & $\mathrm{~V}$ & & & 1 & $\mathrm{~V}$ & & \\
\hline 17. Erigeron canadensis & 2 & $\mathrm{~V}$ & & & 2 & $\mathrm{~V}$ & 2 & $\mathrm{~V}$ & 2 & $\mathrm{~V}$ \\
\hline 18. Galium aparine & & & & & & & & & & \\
\hline 19. Helianthus tuberosus & & & & & & & & & & \\
\hline 20. Iva xanthifolia & & & & & & & & & & \\
\hline 21. Lactuca serriola & & & 1 & $\mathrm{v}$ & 1 & $\mathrm{G}$ & & & & \\
\hline 22. Orobanche spp. & & & & & & & & & & \\
\hline 23. Sonchus arvensis & & & & & & & & & & \\
\hline 24. Sorghum halepense & 2 & $\mathrm{G}$ & 1 & $\mathrm{G}$ & 1 & $\mathrm{G}$ & 1 & $G$ & 2 & $\mathrm{G}$ \\
\hline 25. Xanthium strumarium & 2 & $\mathrm{~V}$ & 1 & $\mathrm{~V}$ & 2 & $\mathrm{~V}$ & 2 & $\mathrm{~V}$ & 2 & $\mathrm{~V}$ \\
\hline
\end{tabular}

Table 4 shows information from second shooting of distribution of weed spices at 9 and 22 of August, on second five locations. Most of weeds were in $G$ (phase of fertility) and $V$ (adult plant) phase, while maize plants were in phase 8 (according to BBCH scale). Weed species were seen at almost all location (10-12) with representation mark from 1 to 3 . Weed species Cirsium arvense, Chenopodium album, Agropyrum repens, Amaranthus retroflexus, Convolvulus arvensis, Cynodon dactylon Sorghum halepense, Xanthium strumarium were found at all locations with average representation mark 2.

\section{CONCLUSIONS}

Based on all presented information from two shootings of distribution and representation of most significant weed species in maize crops at ten locations of Nisava district, we can conclude:

IRTIIE Vol. 6, No. 4, 2018 ISSN 1314-8788 (print), ISSN 1314-8796 (online), doi: 10.15547/artte.2018.04.009 


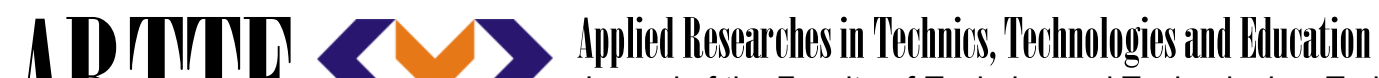 Journal of the Faculty of Technics and Technologies, Trakia University https://sites.google.com/a/trakia-uni.bg/artte/}

- Significant percentage of weed species were found at all ten locations in both shootings;

- In first shooting all present weed species were in phase of mature plant, while their representation was rated with average mark 1, except weed species Cirsium arvense, Cynodon dactylon and Sorghum halepense which had mark 2 on three locations;

- In first shooting perennial weed species had the most coverage of the maize crops;

- Second shooting of the same locations (in August) shown significantly increase of weed species number at all location of Nisava district;

- Most of weed plants were in phase V and $\mathrm{G}$ (adult plant and phase of fertility) in second shooting, with the representation mark from 2 to 3;

- Weed species Agropyrum repens and Cirsium arvense had representation mark of 2 or 3 at all ten locations in second shooting, which means they occupied from $25 \%$ to $50 \%$ of viewed field.

\section{REFERENCES}

[1] Acciares, H. A., Zuluaga, M. S. (2006). Effect of plant row spacing and herbicide use on weed aboveground biomass and corn grain yield. Planta Daninha, 24 (2): 287-293.

[2] Stefanović, L., Tošev, M., Stojšić, S., Vrbničanin, S. (2002). Preliminarni rezultati mapiranja korovske flore u usevu kukuruza. XII simpozijum o zaštiti bilja, Zlatibor, 86.

[3] Stefanović, L., Vrbničanin, S., Malidža, G., Elezović, I., Stanković-Kalezić, R., Marisavljević, D., Jovanović-Radovanov, K. (2006). Kartiranje karantinskih, invazivnih I ekonomski štetnih korova na području Srbije sa predlogom mera suzbijanja. Biljni lekar, XXXIV (3): 195-203.

[4] Simić, M., Brankov, M., \& Dragičević, V. (2017). Effects of nitrogen form, row spacing and herbicide application on weed control and maize biomass production. Herbologia (Sarajevo, BiH), 16.

[5] Vrbničanin, S., Malidža, G., Stefanović, L., Elezović, I., Stanković-Kalezić, R., JovanovićRadovanov, K., Marisavljević, D., Pavlović, D., Gavrić, M. (2009). Distribucija nekih ekonomski štetnih, invazivnih i karantinskih korovskih vrsta na području Srbije. III deo: Prostorna distribucija i zastupljenost osam korovskih vrsta na području Srbije. Biljni lekar, XXXVII (1): 21-30. 